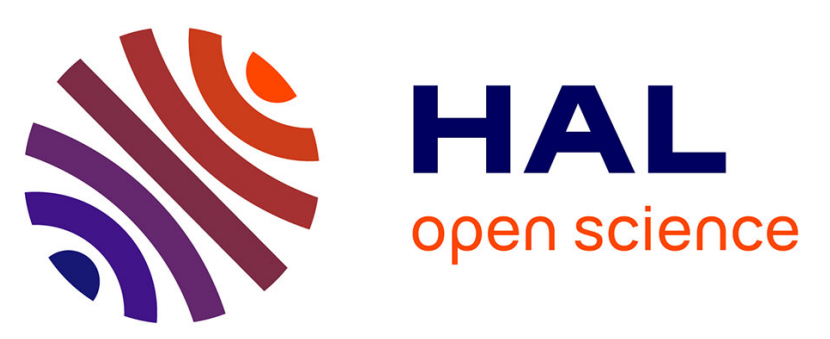

\title{
Le parcours d'interaction sur le web comme expérience d'immersion : entre promesses des interfaces des ONG et mode de perception de l'usager.
}

Sandra Mellot

\section{- To cite this version:}

Sandra Mellot. Le parcours d'interaction sur le web comme expérience d'immersion : entre promesses des interfaces des ONG et mode de perception de l'usager.. "La communication numérique au cœur des sociétés dispositifs, logiques de développement et pratiques", GRESEC, Université Stendhal, Grenoble, May 2015, Echirolles, France. hal-01183205

\section{HAL Id: hal-01183205 \\ https://hal.science/hal-01183205}

Submitted on 7 Aug 2015

HAL is a multi-disciplinary open access archive for the deposit and dissemination of scientific research documents, whether they are published or not. The documents may come from teaching and research institutions in France or abroad, or from public or private research centers.
L'archive ouverte pluridisciplinaire HAL, est destinée au dépôt et à la diffusion de documents scientifiques de niveau recherche, publiés ou non, émanant des établissements d'enseignement et de recherche français ou étrangers, des laboratoires publics ou privés. 


\title{
Sandra Mellot
}

\author{
Le parcours d'interaction sur le web comme expérience d'immersion : \\ entre promesses des interfaces des ONG et mode de perception de l'usager.
}

\section{Colloque international : \\ "La communication numérique au cour des sociétés dispositifs, logiques de développement et pratiques"}

Citation : Sandra Mellot, Le parcours d'interaction sur le web comme expérience d'immersion : entre promesses des interfaces des $O N G$ et mode de perception de l'usager, in Colloque international "La communication numérique au cour des sociétés : dispositifs, logiques de développement et pratiques", 4 mai 2015, Institut des Médias et de la Communication, Échirolles

1 La promesse relationnelle du dispositif

1.1 Préfiguration de la rencontre / configuration de l'expérience.

1.2 De l'expérience d'immersion à sa concrétisation

2. Le Pouvoir de l'usager

2.1 Pouvoir être et Pouvoir faire

2.2 Pouvoir de réalisation ou de résistance

La promesse communicationnelle du numérique s'avère être aujourd'hui avant tout une promesse relationnelle, qui ne se limite pas aux critères de puissance et de rapidité de la mise en relation avec autrui, un environnement, un objet... Cette promesse relationnelle est relative à la qualité du lien d'interdépendance, d'interaction. L'interface devient le lieu de rencontre entre les valeurs de l'instance énonciative et le mode de perception de l'usager. La qualité de cette rencontre est dépendante du processus de co-construction de sens entre l'usager et le dispositif (Pignier, Drouillat, 2005, p.13), processus qui se révèle lors du parcours d'interaction. Nous considérons en effet les interfaces et contenus interactifs «comme partenaires de l'interaction », pourvus d'une «sensibilité réactive » c'est à dire «d'une capacité (prévue lors de sa conception) à réagir très exactement aux impulsions auxquelles nous les soumettons ». (Landowski, 2005, p.41). L'usager doué d'une sensibilité perceptive reçoit cette expérience et agit sur et avec le dispositif en fonction de sa manière de ressentir, de vivre la relation.

Nous nous intéressons aux interfaces des ONG afin de déterminer par quels processus et avec quelle intensité l'usager valorise ou non un parcours d'immersion et ainsi évaluer la qualité de la mise en relation d'un usager avec les valeurs de l'organisation. Nous proposons de déterminer si l'usager s'immerge effectivement dans l'environnement numérique proposé en fonction (i) de la force de l'expérience immersive configurée dans le dispositif et (ii) du pouvoir (de concrétisation, détournement, résistance..) de l'usager en situation d'interaction avec le dispositif immersif.

\section{La promesse relationnelle du dispositif}

\subsection{Préfiguration de la rencontre / configuration de l'expérience.}

Notre analyse à partir des approches communicationnelles des ONG cherche à définir les modalités d'expression de la promesse relationnelle au sein du dispositif numérique. Comment les ONG, en tant qu'instances énonciatives, configurent-elles (au sens de donner un aspect fonctionnel à l'idée) cette relation?

Nous proposons le terme de modèle d'interaction pour définir le mode de configuration du dispositif selon: l'orchestration multimodale de l'interface (l'agencement de différents langages 
visuels, cinétiques, textuels...) et les expériences d'interaction possibles via les fonctionnalités. Nous avons mis en évidence 5 types de modèles d'interaction. Le modèle d'information est fondé sur l'accessibilité, l'efficacité de la mise en relation, permettant à l'usager, par de multiples biais, d'atteindre l'information recherchée (cartes, listes, barres de recherche, images lien ...). Le modèle d'analogie simule un environnement réel dans sa représentation (ressemblance avec le monde environnant) ou/et dans sa perception (création d'un univers polysensoriel...). Le modèle performatif engage un rapport de concrétisation de l'action. L'usager est invité à agir dans ce nouvel espace et d'en éprouver «les effets en retour» (Fogel, Patino, 2013) par exemple en signant une pétition, postant un commentaire ... Le modèle co-créatif permet à l'usager de contribuer à la transformation, à la création d'un nouvel espace. L'usager peut déplacer des éléments, faire apparaître des images, enrichir les contenus, personnaliser une page ... Ces modèles se croisent au sein d'un même dispositif et c'est leur combinaison et la force accordée à chacun qui fixent la stratégie communicationnelle de l'instance énonciative.

Pour que la rencontre se réalise (c'est à dire passe du mode d'existence virtualisé (préfiguré) à celui d'actualisé (configuré) puis de réalisé (vécu) il est nécessaire que l'usager agisse sur le modèle d'interaction et valorise l'expérience contenue dans le dispositif.

Nous avons proposé, en regard des modèles d'interaction, la notion de style d'interaction. Le style d'interaction caractérise cette sensibilité perceptive contenant l'esthésie de l'usager (Pignier, 2012, p.6), sa manière d'appréhender, de ressentir, de prolonger l'expérience médiée par le dispositif. En fonction de son style d'interaction, donc de sa manière de percevoir, l'usager valorise ou dévalorise plus ou moins le parcours contenu dans le modèle d'interaction. Pour analyser un style d'interaction, il est nécessaire d'intégrer à la fois l'étendue de l'action (son étendue spatiale et temporelle) et l'intensité de l'action (sa profondeur). C'est tout l'enjeu de l'analyse sémiotique définie par Jacques Fontanille (Fontanille, 1998, p.71), intégrant le sensible à l'analyse actantielle greimassienne qui portait sur l'aspect structurel de l'enchaînement des actions.

Ainsi, un style d'interaction s'analyse à partir :

- des caractéristiques de l'élément sur lequel agit l'usager (l'agencement des langages et les modes de fonctionnalité), correspondant à l'étendue spatiale d'un style d'interaction.

- la durée de son interaction (étendue temporelle),

- l'aspect itératif, la cohérence et la finalité de son parcours, caractérisant la profondeur des actions et donc l'intensité du style d'interaction.

De la rencontre d'un style d'interaction avec un modèle d'interaction naît une relation coénonciative particulière dont la polarisation plus ou moins positive nous renseigne sur la qualité du processus relationnel engagé. L'analyse du style d'interaction permet ainsi de supposer la désaffection, le penchant ou l'adhésion au modèle d'interaction proposé. On peut alors en déduire une forme d'expérience de l'en acte, expérience de l'activité vivante (évolutive pendant l'interaction) et vécue (à un moment particulier de l'interaction (Fontanille, 2008).

Nous proposons ci-dessous, sous la forme d'un tableau, les expériences de l'en acte possibles en croisant un modèle d'interaction (d'information, d'analogie, performatif, co-créatif) et l'intensité d'un style d'interaction (désaffection, penchant, adhésion). Ainsi, un usager valorisant (en situation de penchant) un modèle d'analogie peut faire l'expérience de la réassurance, du confort, etc.

Tableau 1 : Les expériences de l'interaction en fonction de l'analyse croisée des styles d'interaction avec les modèles d'interaction

\begin{tabular}{|l|l|l|l|}
\cline { 2 - 4 } \multicolumn{1}{c|}{} & Désaffection & Penchant & Adhésion \\
\hline Modèle d'information & $\begin{array}{l}\text { Expérience de l'ennui, } \\
\text { routine }\end{array}$ & $\begin{array}{l}\text { Expérience du } \\
\text { repérage, de la } \\
\text { réflexivité }\end{array}$ & $\begin{array}{l}\text { Expérience de } \\
\text { l'efficacité dans } \\
\text { l'accès }\end{array}$ \\
\hline
\end{tabular}




\begin{tabular}{|l|l|l|l|}
\hline Modèle d'analogie & $\begin{array}{l}\text { Sentiment de déjà-vu, } \\
\text { sentiment de non- } \\
\text { appartenance à l'univers }\end{array}$ & $\begin{array}{l}\text { Expérience de la } \\
\text { réassurance, du confort }\end{array}$ & $\begin{array}{l}\text { Expérience de } \\
\text { l'immersion, } \\
\text { l'appropriation } \\
\text { d'un univers. }\end{array}$ \\
\hline Modèle performatif & $\begin{array}{l}\text { Non implication, } \\
\text { expérience de l'abandon }\end{array}$ & $\begin{array}{l}\text { Expérience de la } \\
\text { contribution }\end{array}$ & $\begin{array}{l}\text { Expérience de la } \\
\text { concrétisation }\end{array}$ \\
\hline Modèle de co-création & $\begin{array}{l}\text { Expérience de la } \\
\text { désorganisation, perte de } \\
\text { repères }\end{array}$ & $\begin{array}{l}\text { Expérience de la } \\
\text { découverte, surprise }\end{array}$ & $\begin{array}{l}\text { Expérience de la } \\
\text { transformation, de } \\
\text { la création }\end{array}$ \\
\hline
\end{tabular}

Nous prolongeons la réflexion de Gian Paolo Caprettini (qui analyse le paysage, sémiotique du monde naturel, en s'inspirant des théories de J.Fontanille), en proposant l'analyse sémiotique d'un monde (pas que) virtuel considérant :

1 Le modèle d'interaction "comme une sémiotique-objet résultant d'une segmentation du monde comportant lui-même, en tant que macro-sémiotique, au moins un principe d'interaction » entre « les énergies » (les actions configurées ) « et les matières » (les orchestrations multimodales).

2 Les styles d'interaction qui résultent « d'un effet de champ perceptif (la prise de position d'un observateur (ici, un usager) qui impose à une portion (de l'interface) un centre de référence, une profondeur, des horizons;»

3 La relation sémiotique (la relation co-énonciative) qui « résulte d'une activité perceptive, et d'une co-présence entre un corps percevant et une portion du monde sensible. » (cf Caprettini G.P, pp.6-7) La relation naît de la « tension entre les modes d'existence (virtuel, actuel, potentiel et réel) projetés sur les prédicats d'expérience » et « les modes d'expérience (devoir, vouloir, croire, savoir, pouvoir) projetés sur les prédicats d'existence » (Caprettini, 2003 p.3)

Ainsi, l'existence virtuelle de l'expérience (contenue dans la configuration du modèle d'interaction) confrontée à l'existence sensible de l'expérience (définie par les styles d'interaction) nous renseigne sur la valorisation ou la dévalorisation de la promesse relationnelle.

\subsection{De l'expérience d'immersion à sa concrétisation}

«L'expérience, [...], ne trouve son sens que dans l'immersion dans le monde. C'est un processus créateur d'objets de valeur (des connaissances, des souvenirs, des compétences, des routines, des empreintes, etc.), dont le critère décisif est l'immédiateté de la relation aux objets, aux situations, au monde en général ; il s'agit d'éprouver les choses, dit Le Robert, et toutes les variantes en diachronie le confirment : faire l'essai de, tenter et risquer, c'est, entre autres et d'abord, se confronter directement aux choses et aux faits. C'est pourquoi on peut définir l'expérience comme production et acquisition de valeurs grâce à l'immédiateté de la relation au monde. » (Caprettini, 2003, p.3).

L'expérience d'immersion, Jean-Jacques Boutaud la décrit en regard des pratiques du Marketing, comme étant le désir d'une société plongée dans «l'hypermodernité » recherchant « la parenthèse « enchantée », sorte d'injonction à vivre pleinement le moment de l'expérience sensible. " Plus que jamais - dit-il - le sens de l'expérience est guidé par la recherche de sensations, par l'immersion dans des contextes enveloppants, polysensoriels. Le régime dominant est donc celui de l'esthésie et des émotions sensorielles favorisées par synesthésie, par coopération des sens. Mais ce paradigme sensoriel s'est développé, dans notre société, à un seuil d'intensité élevé, que l'on qualifie d'hyperesthésie. Rien d'étonnant dans un contexte d'hypermodernité » (Boutaud, 2007).

Nous avons procédé à l'inventaire des modèles d'interaction des ONG configurés dans cette logique immersive et avons observé deux approches qui se couplent dans les dispositifs.

La première approche consiste en la mise en scène d'un univers comme simulacre du terrain d'action de l'ONG ou présentification de ses valeurs. Cette approche est celle configurée dans le modèle d'analogie dont l'expérience d'immersion constitue la valorisation positive de part de l'usager du 
parcours médié par le dispositif (cf Tableau.1). Ces formes de mise en scène du quotidien au sens goffmanien du terme poursuivent un objectif d'immersion de l'usager en ligne dans une atmosphère particulière reliée aux enjeux de l'ONG. Ainsi, l'orchestration multimodale de l'interface permet la création d'un univers polysensoriel censé reproduire ou faire ressentir les conditions de vie ou d'actions liées à la cause de l'ONG.

L'interface d'accueil d'Action-Enfance (http://www.actionenfance.org/) correspond à cet espace simulacre des conditions des enfants dont l'Association s'est donnée la charge d'améliorer le quotidien et d'offrir des perspectives d'avenir. L'agencement de la page joue sur une structuration en unité permettant une rencontre directe avec la seule image proposée celle d'un enfant au centre d'un environnement sombre. L'immersion est provoquée par un univers visuel enveloppant et intrigant, dépourvu d'alternative pour le regard: ou le noir, ou l'enfant au visage éclairé s'offrent à nous. L'image qui semble fixe dans une première perception inattentive est en fait une vidéo dans laquelle l'enfant bouge de gauche à droite très lentement et de manière répétitive. La vidéo est silencieuse. Le signe de la rencontre proposée en première lecture est détourné par le principe d' incommunicabilité signifié par l'attitude de l'enfant et la figuration de son environnement. Une deuxième image vient chasser celle-ci au bout de quelques secondes présentant un point de vue en hauteur actant une forme de distance entre notre espace et celui de l'enfant. L'enfant alors de dos, animé par ce mouvement très lent, se retrouve dans un espace théâtralisé par un jeu de lumière marquant sa position dans l'obscurité et un autre faisceau lumineux suggérant une sortie qui est en fait un mur. Les signifiants de la rencontre (l'enfant seul - la lumière) sont rendus inopérants par leurs propres signifiés (le silence de l'enfant renvoyant à l'incommunicabilité - la lumière artificielle renvoyant à l'absence de sortie réelle). Les signes de présence renvoient par relation afférente à l'absence et réciproquement : présence de lumière - absence de sortie ; présence d'un être - absence de vie sociale (silence et mouvement lent) ; absence de lumière - présence d'une vie ; absence de son - présence d'un mouvement, marquant ainsi l'inadéquation entre l'enfant et son environnement. L'absence de son peut être considérée ici comme une modalité puisque la vidéo suppose de voir mais aussi d'écouter. Ainsi l'orchestration multimodale - visuelle, dynamique (mouvement lent), sonore (par l' absence ) - nous immerge dans l'univers mental de l'enfant afin de reproduire la sensation de malaise. Et le mode textuel «parce que rien n'est joué d'avance » crée un rapport synesthésique car s'entend littéralement. Cette citation se perçoit comme une voix, celle de l'ONG, pour la voix de l'enfant.

La deuxième approche de l"immersion est proposée par le registre de l'action émergeant du modèle d'interaction performatif. Il s'agit cette fois de s'immerger par le geste et par la double concrétisation de ce geste dans le numérique et dans le réel. Le modèle performatif est la simulation d'un espace dans lequel l'usager peut influer sur le cours de l' action proposée. La modalité textuelle, figurée par les boutons jaunes « Donner » et « Agir » et le bouton bleu " Découvrir le clip » différencie deux niveaux d'actions. En bleu une action qui ne sort pas de l'espace numérique : l'usager découvre le clip. L'usager sort ainsi du dispositif immersif refermé sur le malaise de l'enfant pour rejoindre un dispositif immersif ouvert par la présentation d'une vidéo symbolisant une sortie, celle du franchissement du mur par l'enfant. L'action de l'usager a symboliquement ouvert ce passage au sein de l'espace numérique. Les boutons bleus «Agir» ou «Donner» renvoient à des pages de sollicitation de l'usager pour une participation financière ou sur le terrain. Là encore l'action de l'usager permet de passer d'un univers pesant vers la représentation d'enfants souriants manifestant le possible retentissement de l'implication de l'usager. Cette fois-ci l'action est tournée vers le réel : il s'agit de donner pour permettre la sortie de l'enfant de cette impasse que constitue son quotidien. La simulation et stimulation de l'expérience citoyenne par le geste permet à l'usager en ligne d'agir sur cette mise en scène. Il s'agit de «se voir faire» "se voir agir » impliquant un processus de réflexivité à l'œuvre dans les dispositifs immersifs (Boutaud, 2007).

\section{Le Pouvoir de l'usager}

La «communication relation» établie par la stratégie communicationnelle de l'ONG fait ainsi 
émerger une "combinaison d'informations» (Badillo, 2013, p.147) : l'information immersive configurée dans le modèle d'interaction d'analogie et l'information de la concrétisation configurée dans le modèle d'interaction performatif.

Nous avons analysé cette combinaison « immersion-concrétisation » sur plusieurs sites d'ONG afin d'établir une grille d'étude du potentiel de factivité du dispositif, c'est à dire ce que la configuration du dispositif permet à l'usager de vivre, et ce, en fonction du degré d'importance accordé à chacun des modèles d'interaction: d'analogie et performatif.

L'étude de cette combinaison répond à la logique relationnelle des ONG dans l'accompagnement de l'usager comme donateur potentiel. L'immersion correspond aux phases susciter l'intérêt, établir une relation de confiance; La concrétisation correspond à l'étape faire adhérer aux valeurs, aux actions. Cette logique relationnelle retranscrite par le dispositif se définit par une étape d'immersion : du numérique au numérique, puis une étape de concrétisation : du numérique au réel. Ainsi enveloppé dans un univers polysensoriel, l'usager ne doit pas pour autant perdre tous ses repères de la vie réelle afin que le passage à l'acte ne soit pas vécu comme une rupture mais bien comme le prolongement légitime de l'expérience qu'il est en train de vivre.

Quels sont alors les pouvoirs, au sens de capacités accordés par le dispositif, et le pouvoir au sens de puissance d'influence sur le dispositif, dont dispose l'usager?

\section{1 «Pouvoir être» et « Pouvoir faire»}

L'expérience d'immersion configurée dans le modèle d'interaction d'analogie correspond à la capacité pour l'usager d'être, d'exister dans un nouvel espace, d'accueillir une nouvelle représentation de soi comme être dans l'univers de la victime ou être à la place de la victime ou de l'ONG...

L'expérience de concrétisation configurée dans le modèle d'interaction performatif renvoie à la capacité de faire, agir sur la situation numérique ou/et réelle. Ainsi, la combinaison du modèle d'analogie et du modèle performatif est celle du pouvoir être et du pouvoir faire à partir des possibilités offertes par le dispositif et la force de chacun des modèles dans le dispositif. A titre d'exemple, un modèle d'analogie fort associé à un modèle performatif faible implique un pouvoir être fort, pouvoir se figurer dans l'environnement recrée, mais avec un pouvoir faire faible ou inexistant, c'est à dire sans être en capacité d'agir sur l'univers dans lequel l'usager est immergé.

Représenté sur le schéma tensif (Fontanille, 1998) les relations entre pouvoir être et pouvoir faire se distribuent en quatre zones identifiant les possibilités de l'usager en fonction de l'intensité du modèle d'analogie et l'intensité du modèle performatif.

Ill.1 Schéma tensif du «pouvoir être » et « pouvoir faire » configuré dans le dispositif numérique

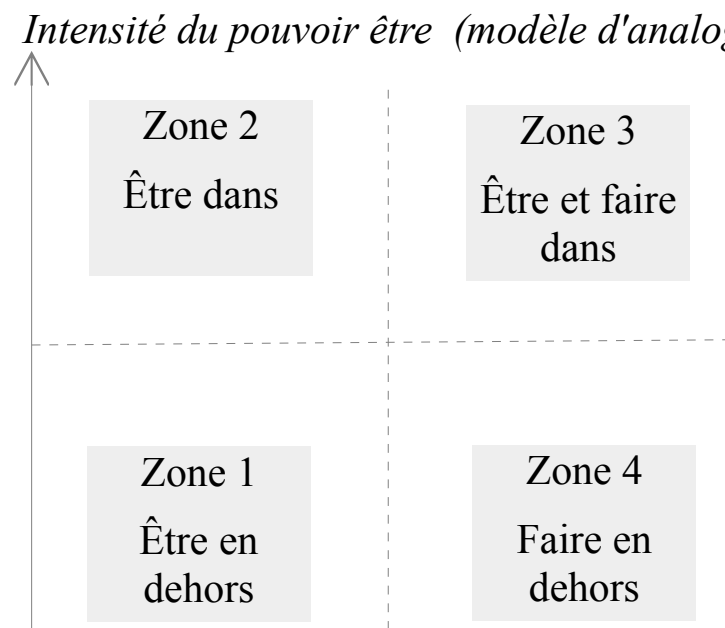




\section{Étendue du pouvoir faire (modèle performatif)}

Zone 1 : ne pas pouvoir être et ne pas pouvoir faire (modèle d'analogie et performatiffaible)

Zone 2 : pouvoir être et ne pas pouvoir faire (modèle d'analogie fort et modèle performatiffaible)

Zone 3 : pouvoir être et pouvoir faire (modèle d'analogie et performatiffort)

Zone 4 : pouvoir faire et ne pas pouvoir être (modèle performatiffort et modèle d'analogie fort)

Pour illustrer ces relations entre pouvoir être et pouvoir faire configurées au sein du dispositif numérique par la combinaison de modèles d'interaction, nous comparons les campagnes sur le web d'Action-Enfance (L'envolée), WWF (Earthours), Reporters Sans Frontières (Voiceless), Solidarités Internationales (Devenez porteur d'eau), Amnesty International (La traversée).

Quand l'interface d'Action-Enfance joue principalement sur le registre du pouvoir être, être en mesure de ressentir le malaise de l'enfant, l'interface proposée par WWF (https://earthhour.paris/) illustre celui du pouvoir faire en proposant à l'usager d'éteindre une lumière de la tour Eiffel virtuellement en s'appropriant une ampoule par un geste (un clic) sur la représentation du monument. La simulation du geste citoyen en faveur de l'économie d'énergie insiste sur la notion de pouvoir contenue dans l'action individuelle virtuelle : « exercez votre pouvoir » (phrase d'accroche de l'interface d'accueil). La translation de ce pourvoir virtuel vers un pouvoir réel lors de l'événement qui eut lieu le 25 mars 2015 éteignant la tour Eiffel durant une heure est la mise en scène réelle de cette première implication dans l'espace numérique.

Le lieu d'immersion et d'action de l'usager se dessine en fonction de la force de chacun des modèles (d'analogie ou performatif). En effet, si l'étendue des possibilités du modèle d'analogie est forte mais celle du performatif faible l'usager sera dans l'espace numérique à vivre (Etre dans) mais s'il souhaite agir, ce sera en dehors de cet espace. Dans ce cas, aucune possibilité n'est configurée dans le dispositif pour concrétiser son immersion.

L'exemple de l'interface mise en place par RSF (Reporters sans frontières) pour la campagne Voiceless (http://www.voiceless-eyes.com/) illustre ce cas de figure. L'usager est invité à se représenter ce que serait l'information sans les reporters. Le dispositif propose une immersion forte par le biais d'une expérience volontaire. Une part de la configuration du modèle d'analogie est visible car l'usager doit intervenir en activant sa webcam et en restant immobile le temps de la reconnaissance de son visage par le dispositif. Lorsque celui-ci a capturé la position du visage, l'expérience peut commencer. Des images de populations victimes défilent. L'usager est invité à passer sa main devant sa bouche. La détection de ce geste fait apparaître une image trafiquée occultant l'expression du crime. Ainsi, sans la voix des reporters, l'information serait biaisée. L'usager est dans l'action, immergé. Si la configuration du modèle performatif permet ici à l'usager d'agir dans l'espace par le mouvement de son corps, il ne permet pas de concrétiser cette action en dehors. Il s'agit ici d'accentuer la sensation d'immersion : d'être dans l'action, d'être à la place de mais sans « effets en retour sur le réel » (Fogel, Patino, 2013)

Une très forte empreinte du modèle performatif et du modèle d'analogie engage une valeur de responsabilisation plus grande. C'est le registre de l'obligation morale que développe cette stratégie. Il s'agit de devoir faire en étant et agissant dans l'espace (être et faire dans).

Amnesty International réalise une campagne sur les demandeurs d'Asile et la dangerosité de leur traversée en mer (http://www.amnesty.fr/Nos-campagnes/SOS-Europe/Presentation). L'immersion est orchestrée par une vidéo occupant intégralement l'écran et se déclenchant automatiquement. L'usager plonge dans les mers sombres d'une traversée clandestine. Un homme tombe à la mer. L'usager est l'observateur impuissant de la noyade, jusqu'à ce qu'il soit invité à intervenir. En effet, le temps d'immersion par la vidéo est interrompu par la modalité textuelle proposant la signature d'une pétition pour l'amélioration des conditions de traversée. L'action de l'usager en ligne va 
déclencher deux scénarios possibles. Le sauvetage de l'homme si la pétition est signée ou la noyade en l'absence de réaction de l'usager. L'usager agit sur l'univers proposé et pour lequel il a ressenti le cours de l'action. Il est placé dans une situation de devoir faire dans l'environnement recréé.

A l'inverse, si la stratégie communicationnelle de Solidarités internationales lors de sa campagne « devenez porteur d'eau » (http://www.votregouttedeau.org/) contient cette promesse immersive dans l'énoncé par « devenez », les modèles d'interaction et d'analogie sont cependant faibles. La configuration du dispositif est pensée au niveau de l'information et non de l'immersion. L'orchestration multimodale (vidéo, texte, boutons d'action) a vocation d'illustration de l'information. L'action de l'usager est limitée à la signature de la pétition, agissant en dehors d'un environnement immersif.

On repère donc, à partir de la configuration de l'interface, quelle promesse est véhiculée par l'instance énonciative en terme de pouvoir faire ou de pouvoir être de l'usager, pouvoir exercé dans ou hors l'environnement recréé.

L'étude sémiotique permet de replacer les promesses des interfaces des sites sur le schéma tensif du pouvoir être et pouvoir faire déterminant ainsi le potentiel de factivité du modèle d'analogie et performatif.

Ill.2 Schéma tensif du " pouvoir être » et " pouvoir faire » configuré dans les dispositifs numériques des $O N G$

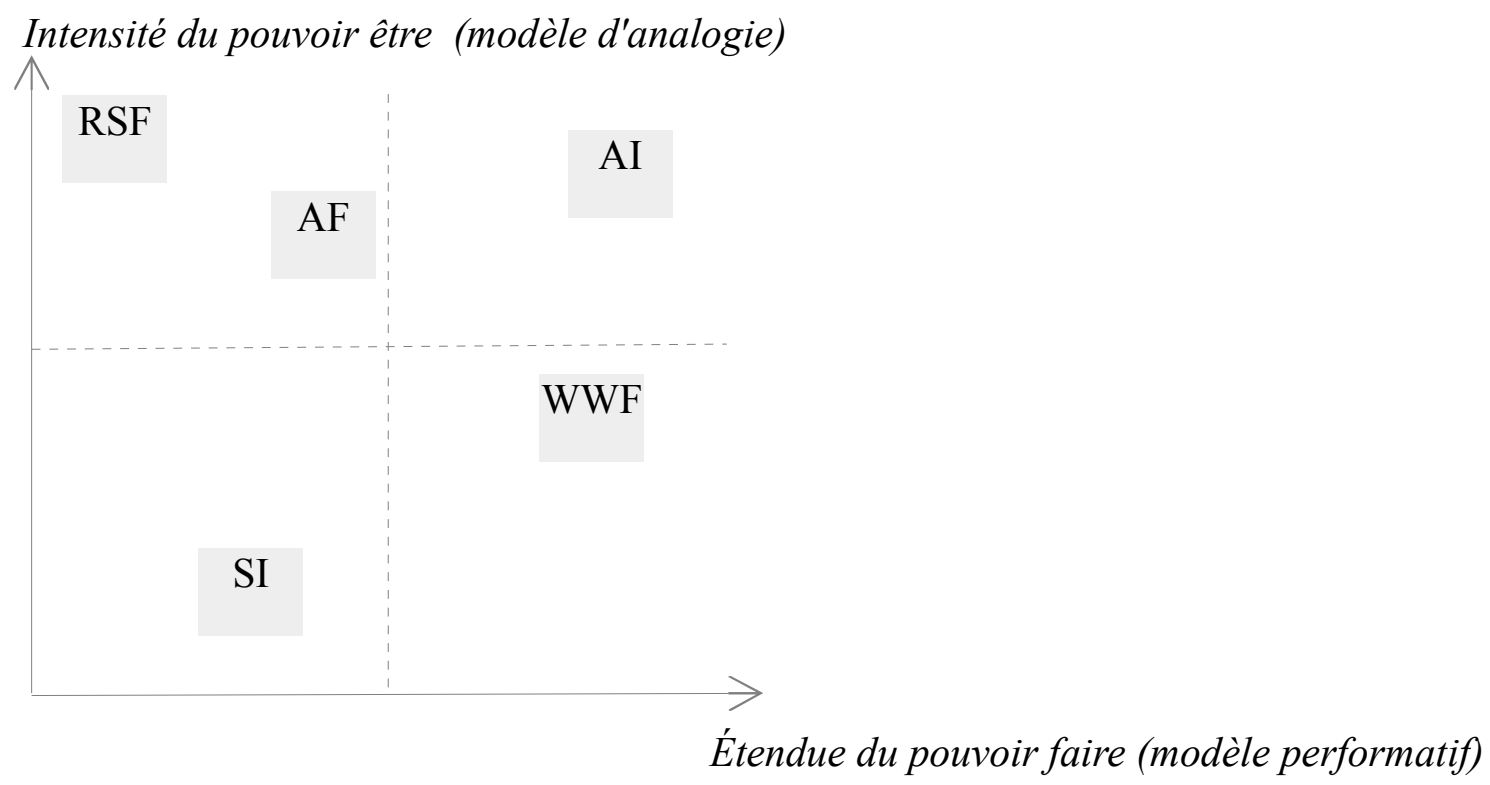

Nous proposons ici le résumé de cette étude à partir des quatre campagnes analysées :

\begin{tabular}{|l|l|l|l|}
\hline Organisations & Modèles d'interaction & Promesses du dispositif & Potentiel de factivité \\
\hline Action enfance (AF) & Modèle d'analogie & $\begin{array}{l}\text { Pouvoir être à la place } \\
\text { de l'enfant victime }\end{array}$ & $\begin{array}{l}\text { Immersion dans les } \\
\text { valeurs d'Action } \\
\text { Enfance }\end{array}$ \\
\hline WWF & Modèle performatif & $\begin{array}{l}\text { Pouvoir faire éteindre } \\
\text { la Tour Eiffel }\end{array}$ & $\begin{array}{l}\text { Concrétisation réelle de } \\
\text { son implication dans } \\
\text { l'espace numérique }\end{array}$ \\
\hline RSF & $\begin{array}{l}\text { Modèle d'analogie } \\
\text { fort / Modèle } \\
\text { performatif contraint }\end{array}$ & $\begin{array}{l}\text { Pouvoir faire } \\
\text { l'expérience d'être dans } \\
\text { un monde sans }\end{array}$ & $\begin{array}{l}\text { Immersion dans l'action } \\
\text { de RSF }\end{array}$ \\
\hline
\end{tabular}




\begin{tabular}{|l|l|l|l|}
\hline & information & \\
\hline $\begin{array}{l}\text { Amnesty International } \\
\text { (AI) }\end{array}$ & $\begin{array}{l}\text { Modèle d'analogie } \\
\text { fort / Modèle } \\
\text { performatif fort }\end{array}$ & $\begin{array}{l}\text { Devoir agir pour le } \\
\text { sauvetage d'un } \\
\text { demandeur d'Asile }\end{array}$ & $\begin{array}{l}\text { Concrétisation } \\
\text { numérique de son } \\
\text { immersion dans les } \\
\text { valeurs de l'ONG }\end{array}$ \\
\hline $\begin{array}{l}\text { Solidarités } \\
\text { internationales (SI) }\end{array}$ & $\begin{array}{l}\text { Modèle performatif } \\
\text { contraint / Modèle } \\
\text { d'analogie faible }\end{array}$ & $\begin{array}{l}\text { Pouvoir agir pour un } \\
\text { accès à l'eau potable }\end{array}$ & $\begin{array}{l}\text { Information pour agir } \\
\text { en dehors de l'espace } \\
\text { numérique }\end{array}$ \\
\hline
\end{tabular}

\subsection{Pouvoir de réalisation ou de résistance}

Ainsi présenté nous avons le résultat du potentiel d'expression de la communication-relation configurée par la combinaison de modèles d'interaction. Ce potentiel est ensuite à confronter avec les parcours d'interaction des usagers. En effet, la valorisation ou dévalorisation de ce potentiel est soumise au style d'interaction de l'usager, à l'intensité avec laquelle il se meut dans l'espace à vivre proposé. Le pouvoir être et pouvoir faire dans une valorisation maximale de la part de l'usager aboutit à la figure passionnelle de l'empathie. Il s'agit de créer un lien fort en se plaçant à l'intérieur de la souffrance, de ce qui est éprouvé par l'autre.

Ill.3 Figure de l'empathie sur le schéma tensif du pouvoir être et pouvoir faire

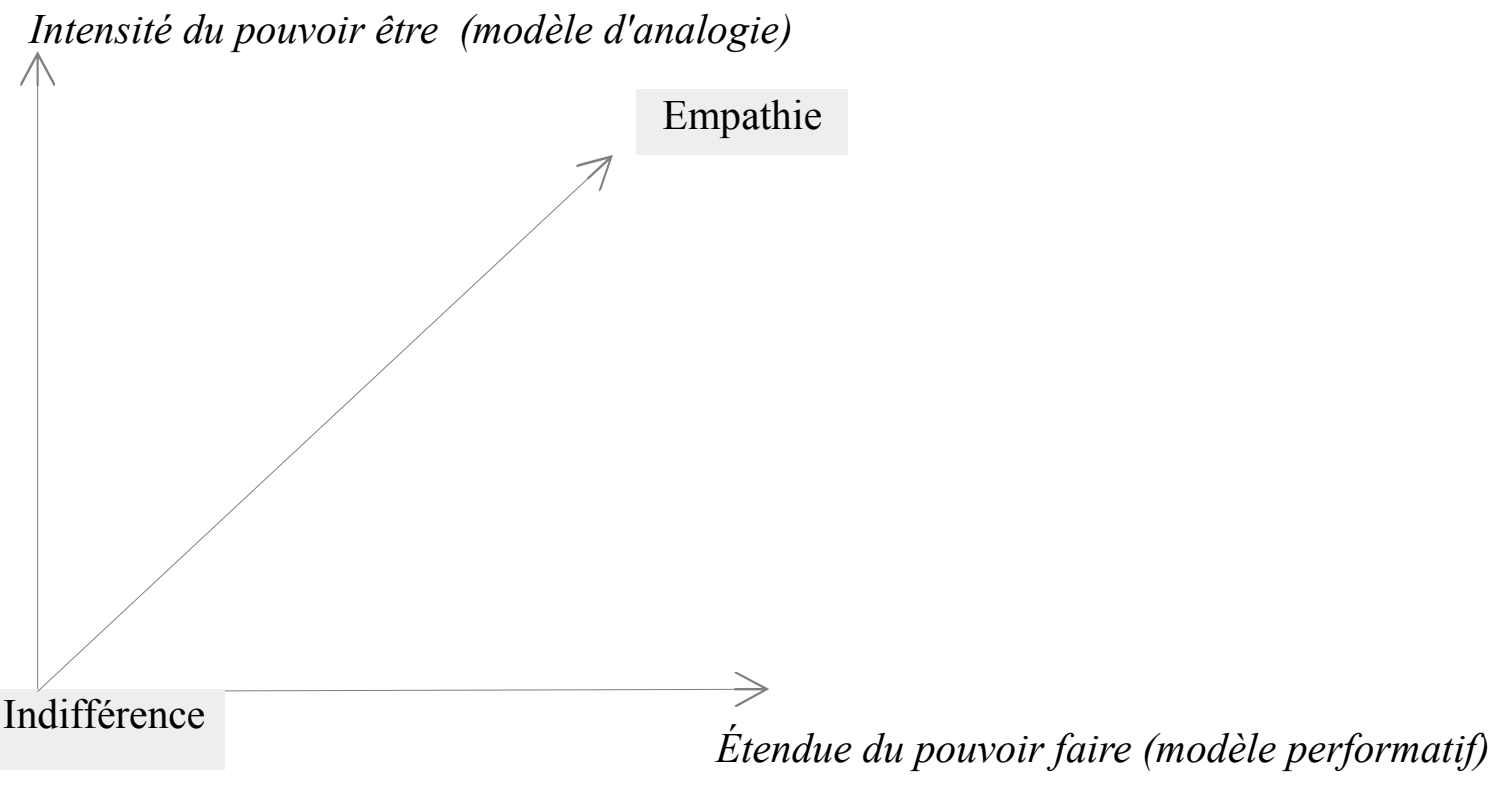

L'usager a donc le pouvoir d'entrer en empathie avec ou de rester en dehors, indifférent aux causes défendues par l'ONG. Nous considérons l'importance de ce que (Paganelli, 2012 p.126) définit comme étant des «stratégies individuelles », c'est à dire des stratégies développées par les usagers pouvant contourner le dispositif ou le détourner de sa finalité.

Céline Paganelli a mis en évidence, dans ses travaux sur les pratiques informationnelles dans le contexte professionnel, la nécessité d'envisager «l'articulation entre des usages et des pratiques sociales développés par les acteurs d'une part et des logiques de production, de conception, en lien notamment avec les politiques publiques et les stratégies d'organisation d'autres part » (Paganelli, 2012, p.137). Nous ne prenons cependant pas en compte les notions qui sont en lien avec les 
taches professionnelles comme celle de niveau d'expertise de l'usager. Les stratégies individuelles sont pour nous la corrélation entre les objectifs de l'usager et les moyens qu'il met en œuvre pour y parvenir. Ces objectifs sont lisibles dans le contenu des pages visitées (contenu informatif, témoignage, pétitions ...) et de l' action finale de l'usager (don, signature, adhésion) . Les moyens mis en œuvre sont identifiés par le parcours d'interaction. Le style d'interaction contient ces contournements et détournements signalés par Pagnelli. Notons, par exemple que le téléchargement fréquent d'images sur les sites d'ONG sont une appropriation personnelle qui peut-être à des fins de contemplation, ce qui ne correspond pas directement à la stratégie d'adhésion ou de valorisation mise en place par l'Organisation. Ce type d' usage ne rentre pas en contraction avec les valeurs mais ne répond pas directement à la promesse communicationnelle de l'ONG.

Jacques Fontanille (Fontanille, 2008) met en évidence le principe d'ajustement, d'accommodation de la pratique au cours d'une situation en fonction de la stratégie de l'instance énonciative et Nicole Pignier souligne les possibles résistances de l'usager à ces stratégies (Pignier, 2012).

Ainsi, le degré de valorisation de l'usager du modèle d'interaction peut prolonger ou altérer l'expérience. La tension entre l'étendue du modèle, c'est à dire l'étendue des possibilités d'interaction, et l'intensité avec laquelle l'usager valorise ce modèle montre les catégories d'expérience sensible. Ainsi l'usager vit l'expérience d'immersion lorsqu'il agit avec l'ensemble des possibilités offertes par le modèle d'interaction d'analogie. L'usager est dedans, dans l'espace à vivre proposé. Un modèle d'analogie fort mais avec une valorisation faible de la part de l'usager implique une submersion pour l'usager. Celui se retrouve en dessous des possibilités configurées dans le dispositif, et est ainsi submergé (conséquence négative de l'immersion) par l'univers proposé. Un modèle faible avec une valorisation forte place l'usager au dessus. Il émerge par rapport à la capacité réactive du modèle et peut donc être frustré du peu de possibilités offertes. Une intensité et une étendue faible revient à laisser l'usager en dehors. Il ne peut pénétrer dans un univers qui n'existe pas.

\section{Ill.5 Schéma tensif de l'expérience d'immersion dans le dispositif numérique}

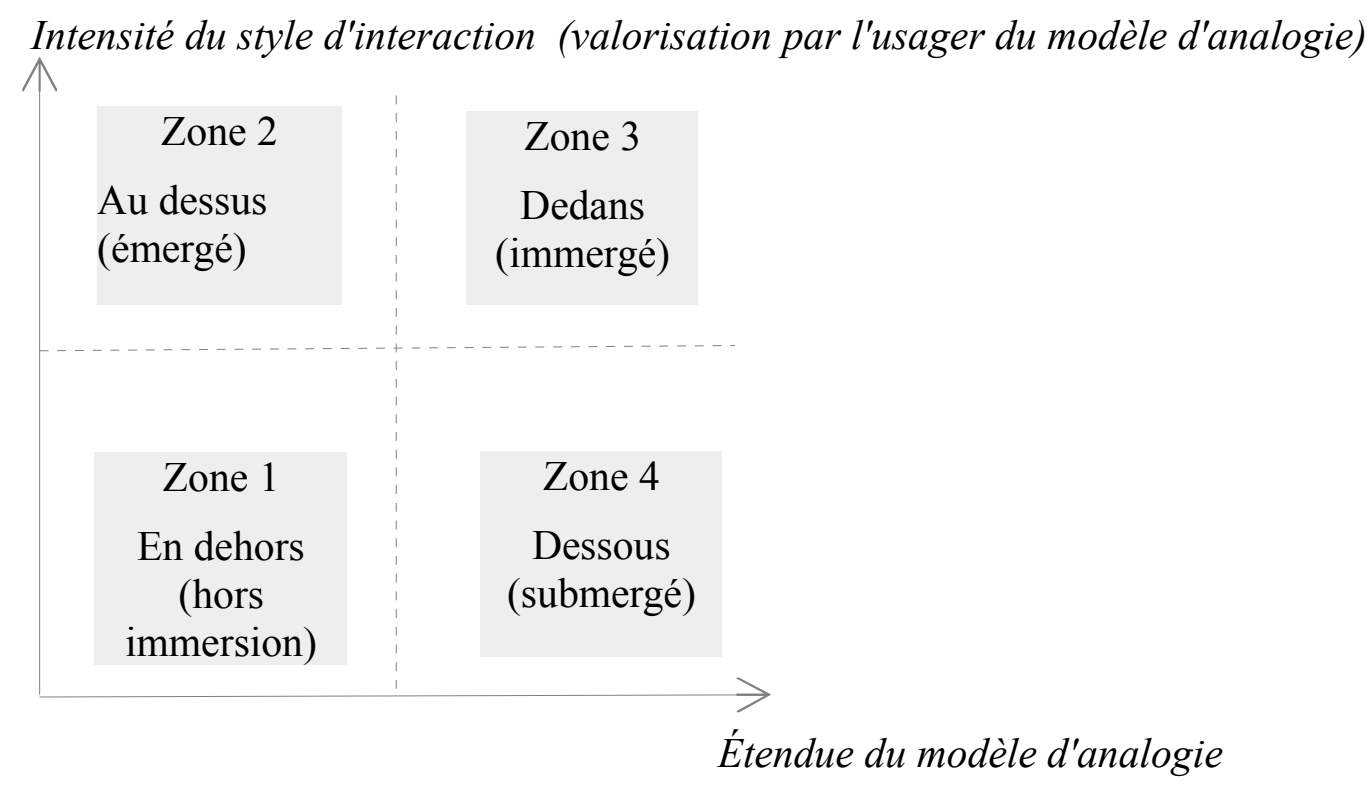

Prenons l'exemple de l'interface de RSF, Voiceless. L'usager s'appropriant le dispositif se retrouve à vivre une expérience d'immersion dans l'action de RSF. Nous considérons que ce modèle contient un fort potentiel (étendue du modèle). Cependant, si l'usager est découragé par le dispositif à mettre en place ou n'a pas tous les outils pour être dans l'expérience (la caméra) l'usager peut-être submergé c'est à dire en dessous des possibilités du modèle. La présence du dispositif peut-être 
ressenti comme trop forte, trop visible pour que le processus de l'expérience ne se réalise. Si l'usager décide de ne pas activer l'expérience dans sa configuration interactive du fait d'un dysfonctionnement du dispositif, d'un matériel absent (la caméra) ou par manque de temps, l'interface de RSF propose une alternative qui est le défilement des images montrant l'information réelle puis l'information trafiquée. Les possibilités d'immersion deviennent faibles, si l'usager est en attente et manifeste une intensité forte, il se situera cette fois au dessus du dispositif et l'expérience configurée sera affaiblie.

Le même cadre de raisonnement appliqué au modèle performatif montre une expérience de réalisation au sens de concrétisation lorsque le modèle performatif est fort et que l'usager en valorise les possibilités d'interaction. A l'inverse le mode de perception de l'usager peut être déréalisant lorsque le modèle performatif est très fort et que l'usager résiste à la sollicitation. Une sollicitation trop faible de l'action de l'usager peut l'inviter à sur-réaliser c'est à dire à chercher des modes d'actions autres pour pallier au manque de sensibilité réactive du dispositif. Si le modèle performatif est faible et que l'usager ne cherche pas à agir, la concrétisation ne se fait pas et reste dans un cadre irréalisable.

Le cas de la campagne sur les demandeurs d'asile (Amnesty international) présente un modèle performatif fort. La valorisation de ce modèle permet la concrétisation. L'usager se réalise alors en tant que sauveteur. Mais dans le cas d'une non action il y a une forme de déréalisation dans le sens d'altération de la perception positive de l'action figurée par la noyade de l'homme.

Dans le cas de la campagne d'Action-Enfance, l'immersion forte est couplée à un modèle performatif limité. L'intensité du mode de perception de l'usager peut instaurer une sur-réalisation c'est à dire une recherche vaine d'actions à réaliser sur l'interface.

\section{Ill.6 Schéma tensif de l'expérience de réalisation (concrétisation) dans le dispositif numérique}

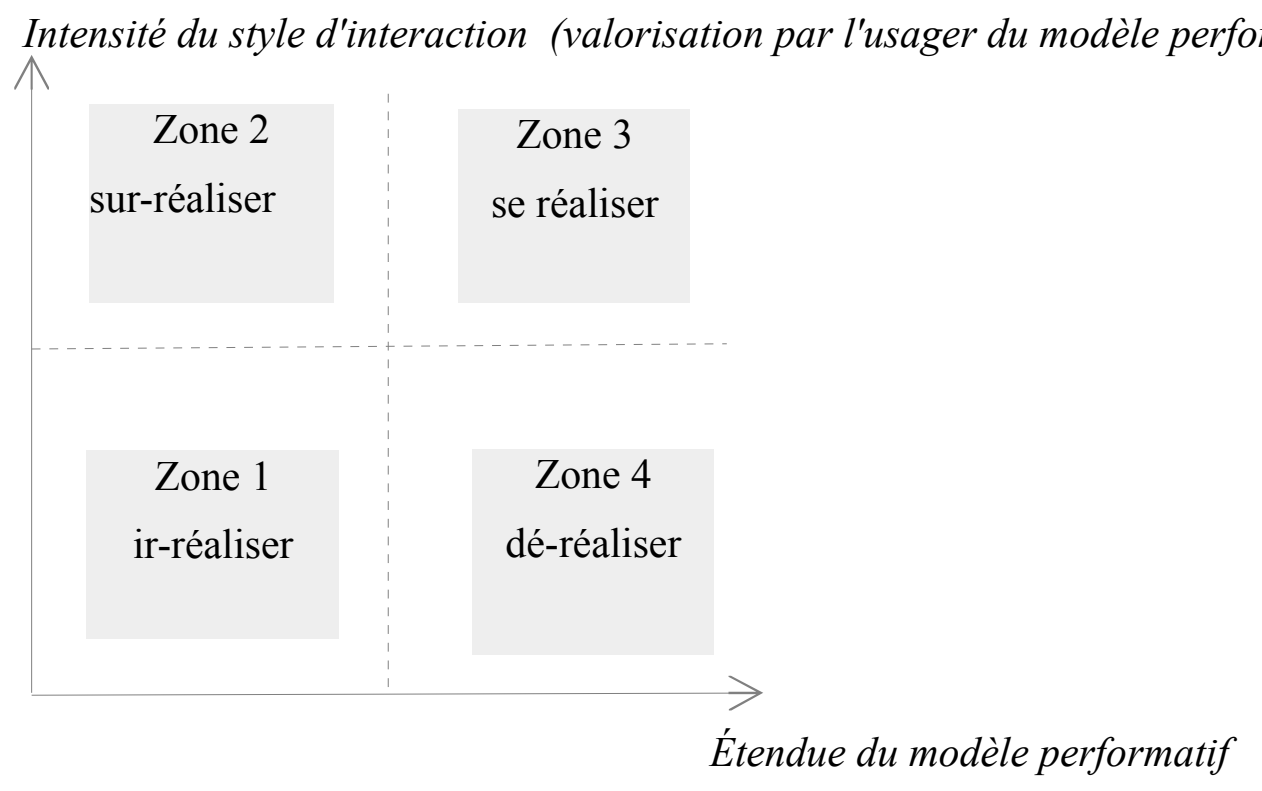

Repérer ces variations d'intensité c'est admettre le caractère mouvant de la relation entre les coénonciateurs et les capacités de l'usager à infléchir une stratégie configurée dans l'interface par la combinaison de modèles d'interaction. Il s'agit donc pour l'Organisation de pourvoir le dispositif d'une sensibilité réactive en capacité d'accomplir la promesse relationnelle. Cette étude contribue à fournir des outils d'analyse des parcours d'interaction en évaluant le potentiel de factivité du dispositif numérique. Ce potentiel dépendant de la configuration du dispositif mais aussi des stratégies développées par les usagers nous avons créé deux catégories d'analyse : modèle d'interaction et style d'interaction permettant une confrontation directe entre les dispositifs et les usages. Cette analyse fixe les enjeux de l'expérience d'immersion au niveau de l'adhésion de 
l'usager aux valeurs de l' ONG. Nous avons proposé la combinaison du modèle d'interaction d'analogie et du modèle performatif pour atteindre cet objectif : le modèle d'analogie permettant à l'usager de ressentir un environnement, d'être dans cet environnement et le modèle performatif permettant d'agir, de concrétiser ses actions au sein de l'environnement. La combinaison du pouvoir être et du pouvoir faire configurée dans ces modèles aboutit à la fabrique de l'empathie médiée par le dispositif, en plaçant l'usager en capacité de vivre et ressentir à la place de l'autre, à l'intérieur de l'environnement de l'autre. Nous pensons que les potentiels ouverts par les technologies numériques souffrent très souvent d'une inadéquation entre les promesses et les modes de perception des usagers, voire de tentation d'uniformisation. C'est pourquoi la relation entre modèle d'interaction et style d'interaction nous apparaît comme essentielle pour réaliser la rencontre entre l'ethos de l'instance énonciative c'est à dire ses valeurs morales et imaginaires (Pignier, Drouillat, 2012, p.50) et le mode de perception de l'usager.

\section{Bibliographie :}

Badillo, Pierre-Yves Badillo (2013), " Les théories de l'innovation revisitées : une lecture communicationnelle et interdisciplinaire de l'innovation ? Du modèle " émetteur " au modèle communicationnel », Les Enjeux de l'Information et de la Communication, n 14/1, p.19-34.

Boutaud, Jean-Jacques (2007), «Du sens, des sens. Sémiotique, marketing et communication en terrain sensible », Semen [En ligne], mis en ligne le 22 août 2007, consulté le 08 avril 2015. URL : http://semen.revues.org/5011

Caprettini Gian Paolo (2003), « Paesaggio, esperienza ed esistenza. Per una semiotica del mondo natural $»$, Semiotiche, $n^{\circ} 1 / 03$, (trad. Française en ligne :

http://www.unilim.fr/pages_perso/jacques.fontanille/articles_pdf/visuel/Paysagesexistenceexperienc e.pdf

Fogel, Jean-François ; Patino, Bruno (2013), La nouvelle condition numérique, Paris : Grasset.

Fontanille, Jacques (1998), Sémiotique du discours, Limoges : Presse Universitaire de Limoges, (collection actes sémiotiques).

Fontanille, Jacques (2008), Pratiques sémiotiques, Paris : Presses Universitaires de France.

Paganelli Céline (2012), Une approche infocommunicationnelle des activités informationnelles en contexte de travail : Acteurs, pratiques et logiques sociales, Université de Grenoble : Library and information sciences, Université de Grenoble, 2012.

Pignier Nicole (2012), « De l'interactivité aux interaction(s) médiatrice(s) », Interfaces numériques, Paris : Lavoisier, $\mathrm{n}^{\circ}$ 1, fev. 2012, 232 p.

Pignier, Nicole ; Drouillat, Benoit (2004), Le webdesign, Sociale expérience des interfaces web, Paris :. Lavoisier.

\section{Auteur :}

Sandra Mellot

Doctorante au Laboratoire CeReS, Universités de Limoges et au département de recherche d'iRaiser, Nantes. sandra@mellot.fr 
Réalise une thèse sous la direction de Nicole Pignier.

Axes de recherche

Pratiques communicationnelles des ONG sur le WEB

Sémiotique du design, des supports et dispositifs numériques

Sémiotique des pratiques d'interaction 